\title{
Association of serum cytokines IL-6, TNF- $\alpha$, PTX-3 and progesterone modulation in cases of recurrent pregnancy loss
}

\author{
Kamal Kishore ${ }^{1}$, Sanjay Sharma ${ }^{2}$, Lavan Singh ${ }^{3}$, Manisha Agarwal ${ }^{4}$, Reema Bhatt ${ }^{5 *}$
}

\author{
${ }^{1}$ Department of Paedtric Surgery, ${ }^{3}$ Department of Pathology, ${ }^{5}$ Army Hospital Research and Referral, Delhi, India \\ ${ }^{2}$ Department of Obstetrics and Gynecology, AFMC Pune, Maharashtra, India \\ ${ }^{4}$ Department of Pathology, Command hospital Eastern Command, West Bengal, India
}

Received: 14 January 2022

Revised: 09 February 2022

Accepted: 10 February 2022

\author{
*Correspondence: \\ Dr. Reema Bhatt, \\ E-mail: reemakamalbhatt@yahoo.co.in
}

Copyright: () the author(s), publisher and licensee Medip Academy. This is an open-access article distributed under the terms of the Creative Commons Attribution Non-Commercial License, which permits unrestricted non-commercial use, distribution, and reproduction in any medium, provided the original work is properly cited.

\begin{abstract}
Background: Cytokines are major immune regulators, which play a critical role in the pathophysiology of unexplained recurrent pregnancy loss. $\mathrm{Th}_{1}$ class of cytokines has been shown to exert deleterious effects on pregnancy causing early loss.

Methods: The changeover in the levels of Th1 cytokines was studied in two major groups; Gp I (N=30) comprised of RPL subjects with two or more consecutive spontaneous miscarriages history and $\mathrm{Gp}$ II $(\mathrm{N}=30)$ comprised of normal pregnancy controls .The efficacy of oral micronized progesterone was assessed in regulation of cytokine levels, with Gp Ia $(\mathrm{N}=15)$ treated with $200 \mathrm{mg}$ oral micronized progesterone and $\mathrm{Gp} \mathrm{Ib}(\mathrm{N}=15)$ comprised of untreated RPL subjects and its role in improving pregnancy outcome was also determined. The present study determined the levels of cytokine TNF- $\alpha$, IL- 6 and PTX-3 in serum samples for all the subjects at three time points at the time of enrolment, with (GpIa) or without (Gp Ib) progesterone administration at 20 weeks or abortion if earlier and at the time of delivery.

Results: The results indicated that the levels of $\mathrm{Th}_{1}$ cytokines (TNF- $\alpha$ [37.80 versus 78.09], IL-6 [16.93 versus 81.12] and PTX-3 [17.42 versus 73.53]) was found to be reduced at every time point in the treated cases with an RPL history as compared to untreated ones. Further, the cases treated with with oral micronized progesterone were found to have better pregnancy outcomes ( $\mathrm{p}<0.005$ significant number of live births).

Conclusions: The levels of interlukins, TNF- $\alpha$, IL-6, PTX-3 in cases of RPL may not have a major contributory role in predicting outcome, however exogenous progesterone decreases the $\mathrm{Th}_{1}$ pro inflammatory response and efficiently improves the pregnancy outcomes by modulation of cytokine levels.
\end{abstract}

\section{INTRODUCTION}

Recurrent pregnancy loss (RPL) is a common clinical reproductive problem, described as the occurrence of 2 or 3 consecutive miscarriages prior to $20^{\text {th }}$ week of the gestation. ${ }^{1}$ The causes of RPLs are diverse including immune, endocrine, parental and fetal genetic and epigenetic factors, metabolic malfunctions, infection factors, anatomic abnormalities and other unknown factors. Despite this, $50 \%$ of the patients who undergo a complete evaluation remain clueless as to the etiology. ${ }^{2}$

Pregnancy is a state of immune suppression. Recently, the contribution of immunomodulatory factors (cytokines) to the etio-pathology of RPL have been recognised. ${ }^{3}$ Immune response in RPL is mainly the imbalance of immune suppression and immune tolerance. $\mathrm{Th}_{1}$ cytokines exert pro-inflammatory effect (TNF- $\alpha$, 
IFN- $\gamma$, IL-2, IL-6), while $\mathrm{Th}_{2}$ cytokines are antiinflammatory in nature. In normal and in late pregnancy, there is a predominance of $\mathrm{Th}_{2}$ cytokines while those in women with pregnancy loss the placental tissue produces $\mathrm{Th}_{1}$ cytokines in early pregnancy. ${ }^{4} \mathrm{Th}_{2}$ cells secrete TGF$\beta$, IL-4, IL-10 that counter regulate $\mathrm{Th}_{1}$ effect and maintain successful pregnancy. $\mathrm{Th}_{2}$ cytokines are important for the maintenance of normal pregnancy. It is hypothesized that failure in the immunological shift from $\mathrm{Th}_{1}$ to $\mathrm{Th}_{2}$ response would lead to the potential harmful effects of $\mathrm{Th}_{1}$ type immune system that may result in pregnancy loss. ${ }^{6}$ The normal range of $\mathrm{Th}_{1} / \mathrm{Th}_{2}$ ratio should be less than $10 .^{5}$

There is a continued quest among the clinicians to find treatment modalities for immunomodulation in RPL patients. Progesterone is one such important steroid hormone that is essential for the maintenance of pregnancy. ${ }^{7}$ A number of studies have indicated that the administration of progesterone prevents the onset of labor, overcomes preterm birth abnormalities and cytokine induced inflammatory response. ${ }^{9}$ Progesterone hormone is responsible for the activation of $\mathrm{T}$-cell immune-suppressive function that modulates proinflammatory cytokine levels. ${ }^{8}$ Progesterone binds to the lymphocyte derived immunomodulatory protein factor receptor on uterine membrane and acts directly or indirectly to modulate the immune system to achieve a successful pregnancy. The present study has been planned to study the effect of micronized progesterone (a pharmacological active supplementation, equivalent to natural progesterone) in RPL patients. The study had two aspects, evaluation of cytokine profile in RPL subjects as compared to normal pregnant women and the efficacy of micronized progesterone in the modulation of aberrant cytokine levels in RPL subjects for better pregnancy outcomes.

\section{METHODS}

\section{Subjects and groups}

The prospective randomized clinical study was undertaken with 30 females with an RPL with history of two or more consecutive miscarriages and 30 females with normal pregnancy, in the department of obstetrics and gynaecology of a tertiary care centre Army hospital research and referral over two years between May 2018 to May 2020.

\section{Inclusion and exclusion criteria}

The inclusion criteria for the selection of subjects included pregnant women of 21-40 year of age, euthyroid females with normal thyroid function tests and history of two or more consecutive miscarriages. Exclusion criteria were women with known causes of abortion and fetus with congenital malformations. All the patients were subjected to, detailed examination for ruling out other causes of recurrent pregnancy loss like thyroid function test, blood glucose level fasting and postprandial, lupus anticoagulant, anticardiolipin antibodies $\operatorname{IgM}, \operatorname{IgG}$ and karyotyping of mother and father, if required protein $\mathrm{C}$, protein $\mathrm{S}$, activated protein $\mathrm{C}$ resistance, antithrombin III, factor 5 Leiden mutation and homocysteine levels were determined. To begin with, the evaluation of baseline cytokine differences among the RPL patients as compared to the normal pregnancy was established at different timelines. Further, the efficacy study of micronized progesterone in the modulation of aberrant cytokine levels in RPL patients for better pregnancy outcomes was performed under two broad groups; group Ia $(\mathrm{N}=15)$ was composed of the subjects with an RPL history of two or more consecutive miscarriages, treated with micronized (200 mg oral) progesterone and group $\mathrm{Ib}$ $(\mathrm{N}=15)$ was of untreated RPL women.

\section{Measurement of cytokines}

Eight milliliters of venous blood were collected in EDTA vacuotainers at three different time points, at enrolment at 20 weeks or abortion (whichever was earlier) and at the time of delivery, from both groups $\mathrm{Gp} 1$ ad $\mathrm{Gp} 2$ mentioned above. Serum samples were used for estimation of cytokine (TNF- $\alpha$ \& IL-6) and pentraxin 3 (PTX-3) levels. Measurement of cytokines and PTX-3 levels was done using an enzyme linked immunosorbent assay (ELISA). The values were estimated using the standard curve of TNF- $\alpha$, IL- 6 and PTX-3. Further, the levels were expressed in $\mathrm{pg} / \mathrm{ml}$.

\section{Statistical analysis}

Each parameter was analyzed statistically by univariate analysis (factorial ANOVA) followed by multiple comparative analysis (Tukey's HSD test/ post hoc test) for different groups.

\section{RESULTS}

\section{Cytokine levels in RPL cases vs normal pregnancy (negative control study)}

The obstetric features and cytokine profiling were analyzed in the women with RPL case $(\mathrm{N}=30)$ as compared to normal pregnant case $(\mathrm{N}=30)$ in similar age matched group of $30.63 \pm 3.89$ years. The RPL group is characterized by the presence of two or more history of miscarriages. The analysis of cytokine showed that the mean amount of $\mathrm{Th}_{1}$ mediators tested for TNF- $\alpha$, IL- 6 cytokines and PTX-3 levels at three-time frames i.e. at enrolment, at 20 weeks or in the event of abortion and at the time of delivery showed notable changes. The levels of TNF- $\alpha$ cytokine were found to be high in RPL subjects with mean values of $57 \mathrm{pg} / \mathrm{ml}, 42.9 \mathrm{pg} / \mathrm{ml}, 41.4 \mathrm{pg} / \mathrm{ml}$ at enrolment, 20 weeks and time of delivery respectively, as compared to healthy subjects (control) with mean value of nearly $6.5 \pm 1.34 \mathrm{pg} / \mathrm{ml}$ of TNF- $\alpha$ at different timelines. Similarly, PTX-3 levels were also found to be higher in women with RPL history with values of $38.7 \mathrm{pg} / \mathrm{ml}, 39.7$ 
$\mathrm{pg} / \mathrm{ml}, 36.1 \mathrm{pg} / \mathrm{ml}$ at enrolment, 20 weeks and at delivery, respectively, as compared to the levels in healthy women $(6.2 \pm 1.56 \mathrm{pg} / \mathrm{ml})$ across different time periods. However, no statistical significant difference was found in the levels of TNF- $\alpha$ and PTX-3 $(p<0.865$ and $p<0.432$, respectively) (Figure 1).

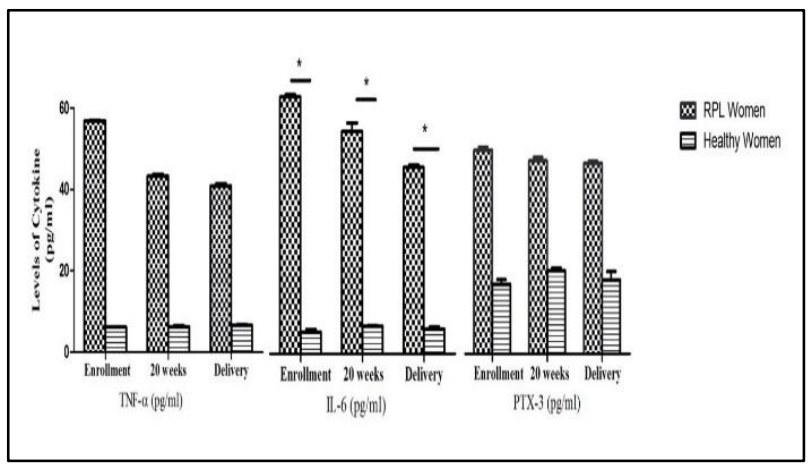

Figure 1: Levels of cytokines at enrolment 20 weeks and at delivery in RPL vs. controls.

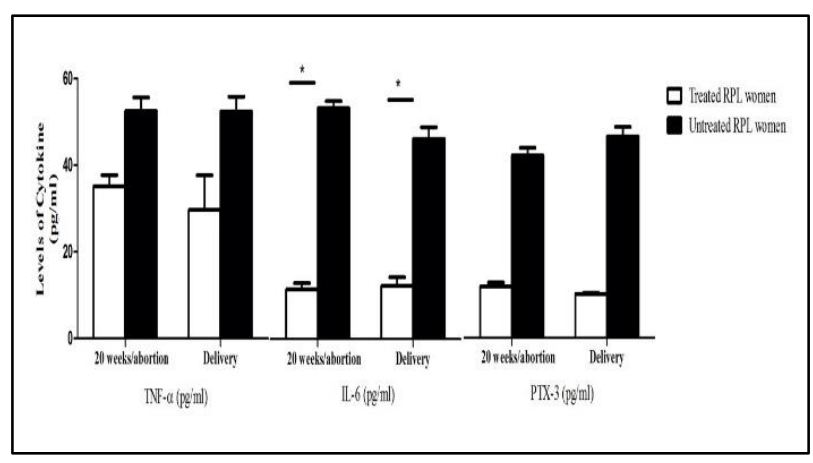

Figure 2: Levels of cytokines in cases of RPL treated with progesterone and untreated cases.

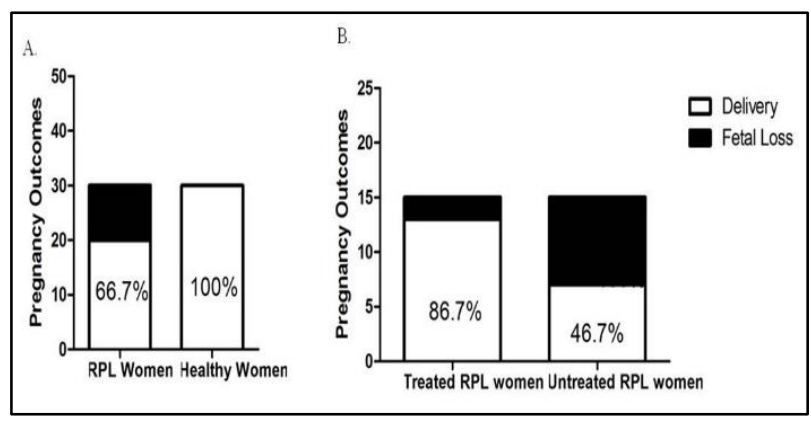

Figure 3: A) pregnancy outcome in RPL women vs. controls, B) pregnancy outcome in RPL treated vs. untreated cases.

Further, other important $\mathrm{Th}_{1}$ cytokine, i.e. IL- 6 levels in the women with RPL history is found with $57.32 \pm 74.20$ $\mathrm{pg} / \mathrm{ml}$ level at enrolment, at 20 weeks was found to be $47.13 \pm 66.50 \mathrm{pg} / \mathrm{ml}$; and $40.27 \pm 60.22 \mathrm{pg} / \mathrm{ml}$ at the time of delivery. The results showed significantly $(* p<0.001)$ high levels of IL-6 amount in RPL subjects as compared to women with normal pregnancy with $6.11 \pm 8.43 \mathrm{pg} / \mathrm{ml}$
IL-6 level (Figure 1). Further, the statistically significant difference in the distribution of pregnancy outcomes, i.e. live birth and fetal loss was also found between the RPL and normal subjects (Figure 3A). The RPL Gp I had more miscarriages due to higher Th1 immunological response as compared to a healthy pregnancy.

\section{Efficacy of micronized progesterone on modulation of aberrant cytokine levels in the RPL cases}

The micronized progesterone has shown to successfully modulate the levels of cytokines and pregnancy outcomes in RPL patients GpIa $(\mathrm{N}=15)$ as compared to untreated GpIb (N=15) RPL women. All the results obtained for cytokine levels was based on the laboratory estimation with respect to two time points, i.e. 20 weeks after drug administration and at the time of delivery, respectively. The cytokine levels among the study participants was not confounded by any factors in the treated women (group Ia) as compared to untreated RPL women (group Ib). Subsequently, the overall mean values of the cytokines TNF- $\alpha$, PTX-3 and IL- 6 were found to be reduced with the micronized progesterone treatment of RPL. The analysis of variance indicated that TNF- $\alpha$ and PTX-3 cytokine levels were found to be decreased at all the time points in the micronized progesterone treated women, however, significant $(\mathrm{p}<0.212)$ change was not found. In contrast, IL-6 levels were found to be significantly $\left({ }^{*} \mathrm{p}<0.003\right)$ decreased and the change remains significant constant at all estimated times (Figure 2). Further, the analysis of pregnancy outcomes revealed significant $(p<0.005)$ increase in the number of live born $(86.7 \%)$ in the micronized progesterone treated group (GpIa) as compared to untreated cases (GpIb) (46.7\%) (Figure 3 B). Such preliminary results indicated the overall improvement in the prevention of abortion with the use of micronized progesterone. Further, correlating the prevention of early miscarriages with the reduction in the levels of Th1 cytokines indicated the treatment efficacy.

\section{DISCUSSION}

Recurrent pregnancy losses are seen in approximately 1$2 \%$ of pregnancies worldwide. It is a challenge for clinical research to look for the critical factors and safe therapeutics to prevent miscarriages during pregnancy. The present study was performed on testing the role of micronized progesterone in cases of RPL. There have been several recommendations on the management of RPL, though the protective effect of micronized progesterone has still not been completely established. 9It has been proposed that non-suppression of Th1 response might turn detrimental for fetal survival. ${ }^{10,11}$ Our data provide indicatory evidence to support the hypothesis and confirms the presence of deviant cytokine profiles in RPL patients. However, the efficacy of micronized progesterone generates a correlation between the immunological modulation and better pregnancy outcome. In normal pregnancy, the cytokines do not act in isolation, but form a complex web of regulatory 
network in which the pregnancy homeostasis is maintained. In RPL, the continuous rise in the levels of Th1 cytokines TNF- $\alpha$, IL- 6 cytokines and PTX-3 has an adverse impact on pregnancy outcome (Figure $3 \mathrm{~A}){ }^{12}$ The mechanism implicates increased levels of prostaglandins which induce and augment uterine contractions and increased levels of oxytocin, leading to miscarriage. Besides, TNF- $\alpha$ and IL- 6 have been directly implicated in promoting apoptosis in uterine tissues via cytokine storm and have a negative effect on the embryo by decreasing the blood flow and causing thrombosis. ${ }^{13,14}$

Previous studies have indicated the effect of IL-6 alone in miscarriages. ${ }^{15}$ Increase in IL- 6 causes immune factor surge response which is critical to pregnancy loss. Besides, increase in PTX-3 is implicated in endothelial dysfunction and uncontrolled immunological complications such as sepsis, vasculitis, preeclampsia and gestational diabetes that affect pregnancy outcome. ${ }^{16}$ Modulation of inflammatory responses is causative in unexplained pregnancy outcomes. The present study established the efficacy of micronized progesterone (200 $\mathrm{mg}$ oral) as treatment modality for the modulation of immunological response and hence favourable pregnancy outcome. We found the decreasing shift in the Th1 cytokine levels in the treated RPL subjects as compared with untreated RPL gp. There was difference in the levels of TNF- $\alpha$ and PTX-3 responses with progesterone treatment in the treated patients as compared to untreated patients though it was not statistically significant. A significant reduction in IL-6 was observed with significant change in the treated group as compared to untreated gp (Figure 2). It has been found that reduction in the cytokine levels by progesterone treatment significantly improves the pregnancy outcomes. Previous studies have also investigated the effect of progesterone on prevention of pre-term birth, spontaneous abortion. ${ }^{17,18}$

\section{Limitations}

The study was underpowered and since sample size was small the results may not be extrapolated in clinical practice like using interlukins for cases of recurrent pregnancy loss. The use of progesterone for recurrent pregnancy loss has been proved by a number of previous studies. Even in our study the results were promising but since sample size was small, we need larger sample size to support our results further.

\section{CONCLUSION}

To conclude, the levels of interlukins, TNF- $\alpha$, IL-6, PTX3 in cases of RPL may not have a major contributory role in predicting outcome, however exogenous progesterone decreases the $\mathrm{Th}_{1}$ pro inflammatory response and efficiently improves the pregnancy outcomes by modulation of cytokine levels. Our study highlights the potential of progesterone as a therapeutic option with the aim to maintain a successful pregnancy outcome.
Funding: No funding sources

Conflict of interest: None declared

Ethical approval: The study was approved by the Institutional Ethics Committee

\section{REFERENCES}

1. Kutteh WH. Recurrent pregnancy loss: An update. Curr Opi Obstet Gynecol. 1999;11:435-9.

2. Krieg S, Westphal L. Immune function and recurrent pregnancy loss. Semin Reprod Med. 2015;33(4):30511.

3. Ksouri H, Zitouni M, Achour W, Makni S, Ben Hassen A. Recurrent pregnancy loss related to immune disorders. Ann Med Interne (Paris). 2003;154(4):233-47.

4. Carp H. Cytokines in recurrent miscarriage. USA: Lupus; 2004:630-4.

5. Carp HJ. Pitfalls in interpreting therapy for early pregnancy. Early Preg Biol Med. 1996;96-101.

6. Wegmann TG. The cytokine bassi for cross-talk between the maternal immune and reproductive systems. Curr Opin Immunol. 1990;2(5):765-9.

7. Mesiano S. Myometrial progesterone responsiveness and the control of human parturition. J Soc Gynecol Invest. 2004;11:193-202.

8. Lei K, Chen L, Georgiou EX, Sooranna SR, Khanjani $\mathrm{S}$, Brosens JJ, et al. Progesterone Acts via the Nuclear Glucocorticoid Receptor to Suppress IL-1 $\beta$-Induced COX-2 Expression in Human Term Myometrial Cells. PLoS One. 2012; 7(11).

9. Evaluation and treatment of recurrent pregnancy loss: A committee opinion. Fertil Steril. 2012;98(5):110311.

10. Bates MD. Aberrant cytokine production by peripheral blood mononuclear cells in recurrent pregnancy loss? Hum Reprod. 2002;17(9):2439-44.

11. Chaouat G, Zourbas S, Ostojic S, Lappree-Delage G, Dubanchet $S$, Ledee N, et al. A brief review of recent data on some cytokine expressions at the maternofoetal interface which might challenge the classical Th1/Th2 dichotomy. J Reprod Immunol. 2002;53(12):241-56.

12. Carp H, Torchinsky A, Fein A, Toder V. Hormones, cytokines and fetal anomalies in habitual abortion. Vol. 15, Gynecol Endocrinol. 2001;472-83.

13. Al Jameil N, Tabassum H, AlMayouf H, Alshenefy A, Almohizea MM, Ali MN. Identification of serum cytokines as markers in women with recurrent pregnancy loss or miscarriage using MILLIPLEX analysis. Biomed Res. 2018;29(18):3512-7.

14. Makhseed M, Raghupathy R, Azizieh F, Omu A, AlShamali E, Ashkanani L. Th1 and Th2 cytokine profiles in recurrent aborters with successful pregnancy and with subsequent abortions. Hum Reprod. 2001;16(10):2219-26.

15. Jenkins C, Roberts J, Wilson R, MacLean MA, Shilito J, Walker JJ. Evidence of a $\mathrm{T}(\mathrm{H}) 1$ type response associated with recurrent miscarriage. Fertil Steril. 2000;73(6):1206-8. 
16. Girardi G, Salmon JE. The role of complement in pregnancy and fetal loss. Autoimmunity. 2003;36:1926.

17. Shah NM, Imami N, Johnson MR. Progesterone modulation of pregnancy-related immune responses. Front Immunol. 2018;9(7):45-9.

18. Ivanova-Todorova E, Kyurkchiev DS, Nalbanski A, Timeva T, Shterev A, Kyurkchiev SD. Production and characterization of a novel monoclonal antibody against progesterone-induced blocking factor (PIBF). J Reprod Immunol. 2008;78(2):94-101.

Cite this article as: Kishore K, Sharma S, Singh L, Agarwal M, Bhatt R. Association of serum cytokines IL-6, TNF- $\alpha$, PTX-3 and progesterone modulation in cases of recurrent pregnancy loss. Int J Reprod Contracept Obstet Gynecol 2022;11:859-63. 